\title{
Comparison of the risk factors and HPV types in males with anogenital warts with and without involvement of the urethral meatus in western Iran
}

\author{
Ali Ebrahimi ${ }^{1}$, Mahmood Reza Moradi², Mansour Rezaei ${ }^{3}$, Hossein Kavoussi ${ }^{1} \bowtie$, Seyed Hamid Madani ${ }^{4}$, Kiarash \\ Mohammadamini ${ }^{1}$, Reza Kavoussi ${ }^{5}$
}

\begin{abstract}
Background: Human papillomavirus (HPV)-induced lesions rarely develop in the urethral meatus (UM); however, their eradication can be problematic.

Methods: A total of 22 patients with anogenital warts (AGWs) in the UM region and 22 patients with AGWs located at other anatomical sites were included in this cross-sectional study. The presence of human papillomavirus (HPV) types in biopsy samples was determined using the HPV Easy typing kit (GenID GmbH, Germany).

Results: Although the patients in the AGW group with involvement of the UM had a higher incidence of reported multiple sexual partners $(63.6 \%$ vs. $40.9 \%)$, larger meatus size ( $81.8 \%$ vs. $36.4 \%)$, and more frequent anal (36.4\% vs. $18.2 \%)$ or unprotected sexual contact $(68.2 \%$ vs. $36.4 \%)$ than those in the AGW group without UM involvement, only the duration of lesions $(p=0.04)$ and meatus size $(p=0.004)$ were significantly different in both groups. Low-risk HPV types were found in $75.0 \%$ and $69.2 \%$ of patients in the AGW groups with and without UM involvement, respectively.

Conclusion: We found that a larger UM size was more prone to the development of meatus AGWs; however, being married, delaying sexual debut, and avoidance of multiple sexual partners seemed to be associated with a decreased risk of development of AGWs, especially within the UM region.
\end{abstract}

Keywords: sexually transmitted infections, urethral meatus warts, HPV typing, prevention

Received: 28 April 2017 | Returned for modification: 23 May 2017| Accepted: 7 August 2017

\section{Introduction}

Anogenital warts (AGWs) are the most common sexually transmitted infections (STIs) (1-3). There are several risk factors for the development of AGWs in men, including sexual contact at a younger age, having multiple sexual partners, being uncircumcised, and having unprotected sexual contact, as well as smoking, low socioeconomic status, and poor education $(4,5)$.

HPV-induced lesions rarely develop in the urethral meatus (UM) and are prone to unsuccessful treatment because of a difficult approach, limitations in safe optional treatment, and fear of complications, especially urethral stenosis. There are few reports regarding susceptibility to the development of AGWs in the UM, in which most of the patients are in their third decade and usually have a previous history of STIs. Urethral instrumentation is also a risk factor, especially in older men, while AGWs with UM involvement are rarely associated with urethral malformations such as hypospadias (6-9).

Because of the increasing incidence of genital HPV infections, the UM being an unusual site for AGWs, the difficulty of eradicating AGWs in this region, and a lack of studies regarding the risk factors of AGWs in the UM, we compared the risk factors and HPV types in males with AGWs with and without UM involvement.

\section{Materials and methods}

\section{Study population}

This cross-sectional study was carried out on 22 heterosexual men with AGWs with involvement of the UM and 22 heterosexual men with AGWs without involvement of the UM at the Hajdaie Dermatology Clinic in Kermanshah, Iran, over a period of 3 years (20132016). Patients whose AGWs with or without involvement of the UM were documented clinically or through histopathological assessment were included in the study. Patients with immunodeficiency, urethral anomaly, recent catheter insertion, or psychological problems, and those that were uncircumcised were excluded from the study.

Demographic data, marital status, sexual habits, number of sexual partners, duration of the disease, and other concurrent STIs were recorded in a questionnaire.

Written informed consent was obtained from every patient. The study was approved by the ethical committee of Kermanshah University of Medical Sciences; the information for all of patients was kept confidential.

\section{Measurement of urethral meatus size}

Assessment of UM size was carried out following appropriate UM treatment and its improvement nearly 1 month after treatment. For measurement of UM size, we used a Nelaton urinary catheter with variable sizes. In patients for whom the Nelaton urinary catheter was easily inserted, a Nelaton urinary catheter equal to or smaller than 18 French (each French is equivalent to $1 / 3$ millimeter) was regarded as a small size, but larger than 18 French was considered a large size for the UM.

${ }^{1}$ Dermatology Department, Hajdaie Dermatology Clinic, Kermanshah University of Medical Sciences (KUMS), Kermanshah, Iran. ${ }^{2}$ Imam Reza Hospital, Kermanshah University of Medical Sciences (KUMS), Kermanshah, Iran. ${ }^{3}$ Health School, Family Health Research Center of Kermanshah University of Medical Sciences (KUMS), Kermanshah, Iran. 4Pathology Department, Kermanshah University of Medical Sciences (KUMS), Kermanshah, Iran. ${ }^{5}$ Kermanshah University of Medical Sciences (KUMS), Kermanshah, Iran. $\bowtie$ Corresponding author: hkavoussi@gmail.com 


\section{Diagnosis of AGWs and other STIs}

In typical clinical presentations, the diagnosis was based on clinical findings, but if there was any doubt in clinical diagnosis then appropriate laboratory assessments such as histopathological or microbiological evaluations were carried out.

\section{Detection and differentiation of HPV types}

Tissue samples of AGWs were collected with a shave biopsy, fixed in $10 \%$ formalin, and subjected to HPV type microarray detection by PCR and subsequent reverse dot-blot hybridization with sequence-specific oligonucleotide probes. We used an HPV Easy typing kit (GenID GmbH, Germany).

Samples were assessed to determine low- and high-risk HPV types. The results of evaluation were reported as high-risk HPV, including HPV 16, 18, 26, 31, 33, 35, 39, 45, 51-53, 56, 58, 66-70, 73, 82, 85, and 97, and low-risk HPV, including HPV 6, 11, 40, 42, 44, and 54. Some HPV types such as HPV 6, 11, 16, 18, 40, and 45 were recognized specifically and reported separately, but other HPV types were reported as a spectrum of high- or low-risk HPV.

\section{Statistical analysis}

First, a one-sample Kolmogorov-Smirnov test (KS) was run to evaluate the normality of quantitative data. Then, based on the results of the KS test, Leven's test and an independent t-test or Mann-Whitney test were applied for normal and abnormal variables, respectively. To compare normal quantitative variables in two groups, we used Leven's test for equality of variances and an independent sample t-test to compare means. To compare abnormal quantitative variables in two groups, we used the Mann-Whitney test. For qualitative variables, we used a chi-squared test. In situations in which a chi-squared test was not possible for $2 \times 2$ tables, Fisher's exact test was used. Finally, the odds ratios (OR) were computed by logistic regression with a $95 \%$ confidence interval (CI). The significance level was considered to be $\leq 0.05$ for test analysis. Analysis of data was carried out using SPSS (version 16) software.

\section{Results}

The mean age of patients was $25.41 \pm 5.48$ years in the AGW group with involvement of the UM and $32.45 \pm 6.58$ years in the AGW group without involvement of the UM (Table 1). All of the patients in both groups had been circumcised due to religious beliefs.

The mean values of duration of lesions in the AGW groups with and without involvement of the UM were $8.95 \pm 3.69$ and $13.09 \pm$ 5.06 months, respectively (Table 1 ). Although there was no statistically significant difference between the two groups in terms of age $(p=1)$, weight $(p=0.55)$, and height $(p=0.1)$, a significant difference was seen in the duration of lesions ( $p=0.04)$ (Table 1). A large meatus size was found in $18(81.8 \%)$ and eight $(36.4 \%)$ of cases in the AGW groups with and without involvement of the $\mathrm{UM}$, respectively ( $p=0.004, \mathrm{OR}=7.875,95 \% \mathrm{CI}=1.964,31.574)$ (Table2). Fourteen (63.6\%) cases in the AGW group with involvement of the UM and nine (40.9\%) cases in the AGW group without involvement of the UM stated that they had had more than one sexual partner during their lifetimes ( $p=0.135, \mathrm{OR}=2.52,95 \% \mathrm{CI}$ $=0.750,8.522$ ) (Table 2 ). Anal sexual contact was reported in eight (36.4\%) and four (18.2\%) patients in the AGW groups with and without involvement of the UM, respectively $(p=0.183, \mathrm{OR}=2.57$, $95 \% \mathrm{CI}=0.641,10.310$ ) (Table 2).
We assessed concurrent STIs in two groups. In the AGW group with involvement of the UM, one (4.5\%), two (9.1\%), and one $(4.5 \%)$ patients had gonorrhea, genital herpes, and molluscum contagiosum, respectively. In the AGW group without involvement of the UM, one (4.5\%) patient had gonorrhea and one (4.5\%) patient had genital herpes.

Of 22 samples in the AGW group with involvement of the UM, HPV infection was detected in eight $(36.4 \%)$ cases, and low-risk HPV types were found in $75.0 \%$ (six of eight patients) and $69.2 \%$ (nine of 13 patients) of patients in AGW groups with and without UM involvement, respectively. The characteristics and details of highand low-risk HPV types in both groups are presented in Table 3.

Table 1 | Demographic data of patients with AGWs with and without involvement of the UM.

\begin{tabular}{|c|c|c|c|}
\hline Variables & $\begin{array}{c}\text { AGWs with UM } \\
\text { involvement }\end{array}$ & $\begin{array}{c}\text { AGWs without UM } \\
\text { involvement }\end{array}$ & $p$ value \\
\hline Mean age (years) & $25.41 \pm 5.48$ & $32.45 \pm 6.58$ & 1 \\
\hline $\begin{array}{l}\text { Mean lesion duration } \\
\text { (months) }\end{array}$ & $8.95 \pm 3.69$ & $13.09 \pm 5.06$ & 0.04 \\
\hline Mean weight (kg) & $71.64 \pm 9.77$ & $73.23 \pm 7.83$ & 0.55 \\
\hline Mean height $(\mathrm{cm})$ & $174 \pm 7.37$ & $171.18 \pm 7.07$ & 0.1 \\
\hline
\end{tabular}

AGWs = anogenital warts, UM = urethral meatus, significance level $p \leq 0.05$

Table 2 | Comparison of risk factors in both groups.

\begin{tabular}{|c|c|c|c|c|}
\hline \multirow[t]{2}{*}{ Variable } & \multicolumn{2}{|c|}{$\begin{array}{l}\text { UM involvement in AGWs, } \\
n(\%), N=22\end{array}$} & \multirow[t]{2}{*}{ OR (95\% Cl) } & \multirow[t]{2}{*}{$p$ value } \\
\hline & With & Without & & \\
\hline $\begin{array}{l}\text { Anal sexual } \\
\text { contact }\end{array}$ & $8(36.4 \%)$ & $4(18.2 \%)$ & $\begin{array}{c}2.57 \\
(0.641-10.310)\end{array}$ & 0.183 \\
\hline $\begin{array}{l}\text { Multiple sexual } \\
\text { partners }\end{array}$ & $14(63.6 \%)$ & $9(40.9 \%)$ & $\begin{array}{c}2.52 \\
(0.750-8.522)\end{array}$ & 0.135 \\
\hline Large meatus size & $18(81.8 \%)$ & $8(36.4 \%)$ & $\begin{array}{c}7.875 \\
(1.964-31.574)\end{array}$ & 0.004 \\
\hline $\begin{array}{l}\text { Unprotected } \\
\text { sexual contact }\end{array}$ & $15(68.2 \%)$ & $8(36.4 \%)$ & $\begin{array}{c}2.941 \\
(0.505-17.141)\end{array}$ & 0.230 \\
\hline Concurrent STIs & $4(18.2 \%)$ & $2(9.1 \%)$ & $\begin{array}{c}2.222 \\
(0.363-13.618)\end{array}$ & 0.388 \\
\hline Marriage & $11(50.0 \%)$ & $5(22.7 \%)$ & $\begin{array}{c}3.4 \\
(0.926-12.489)\end{array}$ & 0.065 \\
\hline
\end{tabular}

AGWs = anogenital warts, $\mathrm{UM}=$ urethral meatus, $\mathrm{OR}=$ odds ratios, $\mathrm{Cl}=$ confidence interval, STIs = sexually transmitted infections, significance level $p \leq$ 0.05

Table 3 | Characteristics of HPV types in both groups.

\begin{tabular}{lcc}
\hline HPV type & $\begin{array}{c}\text { AGW with UM } \\
\text { involvement }\end{array}$ & $\begin{array}{c}\text { AGW without UM } \\
\text { involvement }\end{array}$ \\
\hline HPV genome positive $n$ & 8 & 13 \\
Low-risk HPV $n(\%)$ & $6(75.0 \%)$ & $9(69.2 \%)$ \\
HPV $6 n(\%)$ & $3(50.0 \%)$ & $4(44.4 \%)$ \\
HPV $11 n(\%)$ & $2(33.3 \%)$ & $2(22.2 \%)$ \\
Other low-risk HPV types $n(\%)$ & $1(16.7 \%)$ & $3(33.3 \%)$ \\
High-risk HPV $n(\%)$ & $2(25.0 \%)$ & $4(30.8 \%)$ \\
HPV $16 n(\%)$ & $1(50.0 \%)$ & $2(50.0 \%)$ \\
HPV $18 n(\%)$ & $0(0.0 \%)$ & $1(25.0 \%)$ \\
Other high-risk HPV types $n(\%)$ & $1(50.0 \%)$ & $1(25.0 \%)$ \\
\hline
\end{tabular}

AGWs = anogenital warts, UM = urethral meatus, $\mathrm{HPV}=$ human papillomavirus

\section{Discussion}

To the best of our knowledge, this is the first study to evaluate the risk factors and HPV types in patients with AGWs in the UM region and those with AGWs in other genital areas. The patients in AGW group with involvement of the UM were younger, had multiple sexual partners, and more anal and unprotected sexual contacts; however, these differences were not significantly different compared to the patients in the AGW group without involvement of the UM. 
Vives et al. (6) found most patients with UM warts were in their third decade, although a few senile patients with meatus warts had a previous history of urethral instrumentation. Most studies $(4,5,10,11)$ have reported external AGWs to frequently occur in the third and early fourth decades, which is compatible with the results of our study.

In contrast, there was a significant difference in the duration of lesions between both groups. Shorter duration of lesions and earlier attendance of patients affected with AGWs with UM involvement may be related to tumoral mass sensation in the urethra, dysuria, urethral discharge (12), change in the urine stream (13), and concern for malignancy. In addition, larger meatus size was more commonly observed in the AGW group with involvement of the UM than in the AGW group without involvement of the UM. We tend to think a larger meatus size is associated with more direct contact area during intercourse, thereby increasing the risk of infection.

The most important risk factor for AGWs is the number of lifetime multiple sexual partners $(10,14-19)$. We found that most patients in the AGW group with involvement of the UM and fewer than half of patients in the AGW group without involvement of the UM had multiple sexual partners; however, there were no statistically significant differences between both groups. Our patients in the AGW group with involvement of the UM also more often reported anal sexual contact than those in the AGW group without involvement of the UM, although this observation did not reach statistical significance. Nevertheless, males with external AGWs and a history of anal sexual contact must be evaluated for meatus warts.

In most studies, unprotected sexual contact has been reported as an important factor in AGWs $(10,13,18,19)$. In our study unprotected sexual contact was also a significant factor in the acquisition of genital HPV, especially in the meatus site. We believe protected sexual contact should be an important manner of decreasing meatus AGW involvement.

The incidence of concomitant STIs was comparable between the AGW groups with and without involvement of the UM. Occasionally, genital herpes simplex and molluscum contagiosum were seen with AGWs as coinfection $(11,16,20)$.

In our study, there was no difference in the detection of HPV types between the group of patients with AGWs that involved the UM and those that had AGWs at other locations. Low-risk HPV types were the most commonly detected HPV types $(75.0 \%$ and $69.2 \%$ in the AGW groups with and without UM involvement, respectively). Although low-risk HPV types HPV6 and HPV11 were found to be the causative agents in approximately 90\% of AGWs (21), in a study from Latin America, low-risk HPV6 was found in only $56 \%$ of men with AGWs (22), which is nearly consistent with our findings. These differences may be related to variations in sex, sexual behavior, taking samples from different areas, and the sensitivity and specificity of the HPV genotyping methods. In addition, based on Aguilar et al.'s (8) findings, the isolation of HPV DNA is less frequent in the meatus area than in external AGWs. We think that the low percentage of low-risk HPV types detection may be related to limited detection of our assay to 28 types of HPV. It is also possible that in our area some low-risk HPV types may be more prevalent, which might not be detected by the assay used.

In our study, high-risk HPV types were detected in a significant proportion of patients with AGWs with and without UM involvement. Our results are similar to the observation by Yaghoobi et al. (23), in which HPV16 was the most frequent surface contamination among patients with AGWs. The prevalence of detected highrisk HPV types as surface contamination was also nearly equal in the meatus and external genital areas.

A small sample size, the recruitment of only circumcised males, and a single-center performance were the limitations of our study.

\section{Conclusion}

To conclude, we found that a larger UM size was an important risk factor for the development of AGWs in the UM region; nevertheless, being married, delaying sexual debut, and avoidance multiple sexual partners seemed to be associated with a decreased risk of development of AGWs especially within the UM region. We recommend that further studies be conducted to assess other risk factors and to educate adolescents and young adults regarding STIs and avoidance of high-risk sexual contact. Due to the increasing incidence of AGWs in recent years and difficulty in their eradication, especially in the UM site, an appropriate prevention program should be considered.

\section{References}

1. Azevedo J, Pista A, Lisboa C, Santo I, Azevedo L, Cunhax MJ, et al. Epidemiology of human papillomavirus on anogenital warts in Portugal - the HERCOLES study. J Eur Acad Dermatol Venereol. 2017;31:1342-1348.

2. Yang L, Xie S, Feng X, Chen Y, Zheng T, Dai M, et al. Worldwide prevalence of human papillomavirus and relative risk of prostate cancer: a meta-analysis. Sci Rep. 2015;5:14667.

3. Shigehara K, Sasagawa T, Kawaguchi S, Kobori Y, Nakashima T, Shimamura M, et al. Prevalence of human papillomavirus infection in the urinary tract of men with urethritis. Int J Urol. 2010;17:563-8.

4. Tamer E, Cakmak SK, İlhan MN, Artüz F. Demographic characteristics and risk factors in Turkish patients with anogenital warts. J Infect Public Health. 2016; 9:661-6.

5. Tas B, Turker K, Balci E. Risk-factors and awareness of HPV in Turkish people with anogenital warts in Bagcilar district: a cross-sectional study. Arch Iran Med. 2016;19:715-9.

6. Vives Á, Vazquez A, Rajmil O, Cosentino M. Urethral condylomas in men: experience in 123 patients without previous treatment. Int J STD AIDS. 2016;27:39-43.

7. Takahashi S, Hirano Y, Kawamura T, Homma Y. Intraurethral condylomata acuminata associated with genital piercings. Int J STD AIDS. 2013;24:67-9.

8. Aguilar LV, Lazcano - Ponce E, Vaccarella S, Cruz A, Hernández P, Smith JS, et al. Human papillomavirus in men: comparison of different genital sites. Sex Transm Infect. 2006;82:31-33.

9. Sumino $\mathrm{Y}$, Mimata $\mathrm{H}$, Momura Y. Urethral condyloma acuminata following urethral instrumentation in an elderly man. Int J Urol. 2004;11:928-30.
10. Svare El, Kjaer SK, Worm AM, Osterlind A, Meijer CJ, van den Brule AJ. Risk factors for genital HPV DNA in men resemble those found in women: a study of male attendees at a Danish STD clinic. Sex Transm Infect. 2002;78:215-8.

11. Soori T, Hallaji Z, Noroozi-Nejad E. Genital warts in 250 Iranian patients and their high-risk sexual behaviors. Arch Iran Med. 2013;16:518-20.

12. Berger RE, Lee JC. Genital warts. In: Walsh PC, Retik AB, Vaughan ED Jr, Wein AJ, editors. Campbell's urology. 8th ed. Philadelphia, PA: WB Saunders; 2002. p. 685.

13. Kilciler M, Bedir S, Erdemir F, Coban H, Erten K, Ors O, et al. Condylomata acuminata of external urethral meatus causing infravesical obstruction. Int Urol Nephrol. 2007;39:107-9.

14. Goldstone S, Palefsky JM, Giuliano AR, Moreira ED Jr, Aranda C, Jessen H, et al. Prevalence of and risk factors for human papillomavirus (HPV) infection among HIV-seronegative men who have sex with men. J Infect Dis. 2011;203:66-74.

15. Mueller N, Cruz-Valdéz A, Aguilar LV, Franceschi S, Hernández-Avila M, LazcanoPonce. Determinants of prevalence, acquisition, and persistence of human papillomavirus in healthy Mexican military men. Cancer Epidemiol Biomarkers Prev. 2005;14:1710-614.

16. Shin HR, Franceschi S, Vaccarella S, Roh JW, Ju YH, Oh JK, et al. Prevalence and determinants of genital infection with papillomavirus, in female and male university students in Busan, South Korea. J Infect Dis. 2004;190:468-76.

17. Bakes DM, Snijders PJ, Hudgens MG, Bailey RC, Bogaarts M, Agot K, et al. Sexual behaviour and less frequent bathing are associated with higher human papillomavirus incidence in a cohort study of uncircumcised Kenyan men. Sex Transm Infect. 2013;89:148-55. 
18. Johnson AM, Mercer CH, Beddows S, de Silva N, Desai S, Howell-Jones R, et al. Epidemiology of, and behavioural risk factors for, sexually transmitted human papillomavirus infection in men and women in Britain. Sex Transm Infect. 2012;88:212-7.

19. Baldwin SB, Wallace DR, Papenfuss MR, Abrahamsen M, Vaught LC, Giuliano AR. Condom use and other factors affecting penile human papillomavirus detection in men attending a sexually transmitted disease clinic. Sex Transm Dis. 2004;31:601-7.

20. Fageeh WM. Sexually transmitted infections among patients with herpes sim plex virus at King Abdulaziz University Hospital. BMC Res Notes. 2013;6:301.
21. Hawkins MG, Winder DM, Ball SL, Vaughan K, Sonnex C, Stanley MA, et al. Detection of specific HPV subtypes responsible for the pathogenesis of condylomata acuminata. Virol J. 2013;10:137.

22. Hernandez-Suarez G, Pineros M, Vargas JC, Orjuela L, Hernandez F, Peroza C, et al. Human papillomavirus genotypes in genital warts in Latin America: a crosssectional study in Bogota, Colombia. Int J STD AIDS. 2013;24:567-72.

23. Yaghoobi R, Makvandi M, Afshar N, Pazyar N, Hamidifard M, Sharifpour C. Highfrequency of human papillomavirus genotype 16 among patients with anogenital warts. Jundishapur J Microbiol. 2015;8:e25882. 